\title{
Rebaudioside A Enhances LDL Cholesterol Uptake in HepG2 Cells via Suppression of HMGCR Expression
}

\author{
Amirul Nazhan Ilias ${ }^{1}$, Intan Safinar Ismail ${ }^{2}$, Hazilawati Hamzah ${ }^{3}$, \\ Taznim Begam Mohd Mohidin ${ }^{4}$, Mohd Faiz Idris ${ }^{5}$, Mokrish Ajat*1
}

\begin{abstract}
Background: Rebaudioside A is one of the major diterpene glycosides found in Stevia had been reported to possess anti-hyperlipidemic effects. In this study, we explore the potential cholesterol-regulating mechanisms of Rebaudioside A in the human hepatoma (HepG2) cell line in comparison with simvastatin.

Methods: Cells were incubated with Rebaudioside A at several concentrations $(0-10 \mu \mathrm{M})$ to determine the cytotoxicity by the MTT assay. Cells were treated with selected dosage $(1$ and $5 \mu \mathrm{M})$ in further experiments. Total cellular lipid was extracted by Bligh and Dyer method and subjected to quantitative colorimetric assay. To illustrate the effect of Rebaudioside A on cellular lipid droplets and low-density lipoprotein receptors, treated cells were subjected to immunofluorescence microscopy. Finally, we investigated the expression of experimental gene patterns of cells in response to treatment.

Results: In this study, cytotoxicity of Rebaudioside A was determined at $27.72 \mu \mathrm{M}$. Treatment of cells with a higher concentration of Rebaudioside A promotes better hepatocellular cholesterol internalization and ameliorates cholesterol-regulating genes such as HMGCR, LDLR, and ACAT2.

Conclusions: In conclusion, our data demonstrated that Rebaudioside A is capable to regulate cholesterol levels in HepG2 cells. Hence, we proposed that Rebaudioside A offers a potential alternative to statins for atherosclerosis therapy.
\end{abstract}

Keywords: Rebaudioside A, Anti-hypercholesterolemia, Lipid droplets, Low-density lipoprotein, HMGCR.

\section{Introduction}

An evidence-based phytotherapy pathway that relies on scientific and clinical data is a crucial approach before the establishment of plantbased derivatives in medical practice (1). Stevia rebaudiana Bertoni is an intense, non-nutritive natural sweetener that has been a popular sugar substitute used in various industries as well as phytotherapy (2). Following the first domestication in Japan, large-scale cultivation now exists in other countries to fulfil the economical demands (3). Phytochemical of
Stevia leaves revealed the presence of steviol glycosides, and other important secondary metabolites each with various therapeutic potentials $(3,4)$. To date, there are 11 major steviol glycosides detected in the plant (5).

Rebaudioside A accounted for up to $6.5 \%$ of the total dry weight of the leaf is of interest due to its potent sweetness estimated to be 400 times sweeter than sucrose (6). There exist quite a lot of discussion as to therapeutic applications of Rebaudioside A (7-10). Recent investigations

1: Department of Veterinary Preclinical Science, Faculty of Veterinary Medicine, Universiti Putra Malaysia, 43400 UPM, Selangor, Malaysia. 2: Department of Chemistry, Faculty of Science, Universiti Putra Malaysia, 43400 UPM, Selangor, Malaysia.

3: Department of Veterinary Pathology and Microbiology, Faculty of Veterinary Medicine, Universiti Putra Malaysia, 43400 UPM, elangor, Malaysia.

4: Institute of Biological Sciences, Faculty of Science, University of Malaya, 50603 Kuala Lumpur, Malaysia.

5: Pusat Bahasa dan Pengajian Umum, Universiti Pendidikan Sultan Idris, 35900 Tanjong Malim, Perak, Malaysia.

*Corresponding author: Mokrish Ajat; Tel: +603 97693419; E-mail: mokrish@upm.edu.my.

Received: 13 Jan, 2021; Accepted: 8 Feb, 2021 
demonstrated the potential role of Rebaudioside $\mathrm{A}$ as a hepatoprotective agent $(11,12)$. More importantly, it has been shown that an improvement in the lipid profile of experimentally induced diabetic rats can be achieved by oral administration of this diterpenoid glycoside (13) in which further support the notion that Rebaudioside A possesses potential lipid-regulating activity.

Atherosclerosis is a chronic inflammatory disease affecting the intima of medium and large-sized arteries, likely to occur at arterial bifurcations $(14,15)$. The disease has been recognized as the principal cause of clinically important cardiovascular diseases (CVDs) (16). Pioneering work on atherosclerosis has been carried out earlier by Alexander Iosifovich Ignatowski in 1907 in which he provided evidence of aortic atherosclerotic lesion in experimental rabbits fed with various types of animal proteins (17). Conceptually similar work has also been carried out by Anichkov and Semen Chalatov in 1912 that extends the idea of cholesterol in atherosclerosis development (17). In addition to non-pharmacological approaches, few medications have been developed to intervene with abnormal plasma cholesterol levels (18). A class of cholesterol-modifying medications known as statins is commonly prescribed to hyperlipidemic patients (18). Likewise, considering that reactive oxygen species (ROS) and oxidative stress initiate atherosclerosis which cause coronary heart disease, antioxidants are beneficial to prevent such events (19). Despite the efficiency of statins in lowering cholesterol, many side effects have been discussed (20-22) thus further motivates the need for an alternative to replacing statins. Therefore, in this study, we are interested in examining the unexplored cholesterol-lowering potential of Rebaudioside A in the HepG2 cell line.

\section{Materials and Methods}

\section{Cell culture}

HepG2 cells (ATCC number: ATCC ${ }^{\circledR}$ HB$8065^{\mathrm{TM}}$ ) were grown in $90 \%$ advanced minimum essential media (AMEM) supplemented with $10 \%$ fetal bovine serum
(FBS), $1 \%$ penicillin-streptomycin and $1 \%$ amphotericin-B. All cell culture reagents were from Gibco (USA). Cells were maintained in a humidified incubator with $5 \% \mathrm{CO}_{2}$ at $37{ }^{\circ} \mathrm{C}$.

\section{Preparation of Rebaudioside A and Simvastatin} HPLC grade of Rebaudioside A $(\geq 96 \%)$ and Simvastatin $(\geq 97 \%)$ were from Sigma Aldrich (USA). Rebaudioside A (RA) was prepared as a stock solution in a sterile dimethyl sulfoxide with a final concentration of $1 \mathrm{mM}$. Simvastatin was activated as mentioned by (23). Both solutions were stored at $4{ }^{\circ} \mathrm{C}$ until further analysis.

\section{Cell viability assay}

Cells $\left(1 \times 10^{4}\right.$ cells per well) were incubated in 96 well-plates for 24 hours. The following day, the culture medium was removed and cells were further incubated with RA $(0-10 \mu \mathrm{M})$ overnight. Upon 24 hours, the cytotoxic effect was determined by the MTT assay (24). The halfmaximal inhibitory concentration $\left(\mathrm{IC}_{50}\right)$ was determined by graphically plotting the value of cell viability (\%) accordingly:

Cell viability $(\%)=($ Mean Treated OD (A570) Mean Background $\left.\mathrm{OD}_{(\mathrm{A} 570)}\right)$ / (Mean Control $\mathrm{OD}_{(\mathrm{A} 570)}$-Mean Background $\left.\mathrm{OD}_{(\mathrm{A} 570)}\right)$ x 100

\section{Cell treatment}

For cholesterol quantification and cDNA expression analysis cells ( $3 \times 10^{5}$ cells per well) were seeded in 6 well-plates and for immunofluorescence microscopy $\left(1 \times 10^{3}\right.$ cells per glass coverslip) in 4 well-plates. Cells were treated for 24 hours accordingly: 1) Base control (BC) AMEM and FBS only; 2) Negative control (NC) AMEM, FBS, CD lipid concentrate, 50X cholesterol lipid concentrate, and human plasma LDL; 3) Positive control (PC) AMEM, FBS, CD lipid concentrate, 50X cholesterol lipid concentrate, and human plasma LDL with $10 \mu \mathrm{M}$ simvastatin; 4) Low dosage RA (LRA) AMEM, FBS, CD lipid concentrate, 50X cholesterol lipid concentrate, and human plasma LDL with $1.0 \mu \mathrm{M}$ RA; and 5) High dosage RA (HRA) AMEM, FBS, CD lipid concentrate, 50X cholesterol lipid concentrate, and human plasma LDL with 5.0 $\mu \mathrm{M}$ RA. For cholesterol quantification and 
cDNA analysis, the treatment medium was removed, cells were washed with PBS, scraped off, and collected into a $1.5 \mathrm{ml}$ tube. For immunofluorescence imaging, cells were stained, mounted on coverslips, and viewed.

\section{Cholesterol quantification and protein determination}

Before analysis, cells were homogenized for 2 minutes at $20 \%$ amplitude on ice using an ultrasonic dismembrator (Thermo Fisher Scientific, USA) followed by lipid extraction according to Bligh and Dyer method (25). Total cellular cholesterol (TCC) was quantified by Cholesterol Liquicolor Kit (Human Diagnostic, USA) according to the manufacturer's instruction at 540nm wavelength using a spectrophotometer. TCC was calculated as follow:

Cholesterol concentration $(\mathrm{mmol} / \mathrm{L})=5.17 \mathrm{x}$ [Mean Treated $\mathrm{OD}_{(\mathrm{A} 540)} /$ Average Blank $\mathrm{OD}_{(\mathrm{A} 540)]} /\left[\right.$ Average Standard $\mathrm{OD}_{\left(\mathrm{A}_{540)} / \text { Average }\right.}$ Blank OD $(\mathrm{A} 540)]$

Protein concentration was determined using the Pierce ${ }^{\mathrm{TM}}$ BCA Protein Assay Kit (Thermo Fisher Scientific, USA). Data acquired from protein determination was used to normalize data of cholesterol quantification analysis.

\section{Immunofluorescence microscopy}

Qualitative expression of the nucleus $(\mathrm{N})$, lipid droplets (LDs), and low-density lipoprotein receptors (LDLR) were observed by immunofluorescence microscopy. Cells were fixed with $4 \%$ paraformaldehyde (Sigma Aldrich, USA) in PBS for 60 minutes at room temperature. The fixative agent was discarded and cells were washed with PBS three times, each for 5 minutes. Afterward, cells were submerged in a concoction of $2 \%$ BSA (Amresco, USA) to block non-specific binding and $0.2 \%$ Triton X-100 (Sigma Aldrich) to aid cellular permeabilization. Then, cells were incubated with primary antibody against LDLR (1:50, ab175883, Abcam, UK) for 1 hour. Counterstaining was carried out using fluorescently tagged Alexa Fluor ${ }^{\circledR} 594$ Donkey Anti-Rabbit IgG [H+L] secondary antibody (1:200; Thermo Fisher Scientific, USA), Bodipy 493/503 (1:50; Abcam, USA) and Hoechst 33342 (1:50; Gibco, USA). Finally, the coverslip was mounted onto a glass slide with FluorSave $^{\mathrm{TM}}$ Reagent (Sigma Aldrich, USA) and viewed at 200X magnification using an inverted fluorescence microscope (Nikon Eclipse Ti-S, Japan).

\section{RNA isolation and optimization of primers}

RNA was extracted using the RNeasy Mini Kit (Qiagen, Germany) as mentioned in the manual. The purity and quantity of total cellular RNA were assessed spectrophotometrically using a NanoQuant Plate ${ }^{\mathrm{TM}}$ (Tecan, Switzerland). Optimization of experimental primers (IDT, Singapore) in Table 1 with standardized total RNA (100 ng/ $\mu \mathrm{l})$ was carried out by conventional reverse transcriptase-polymerase chain reaction (RT-PCR) using the Access RTPCR System master mix (Promega, USA) in the Thermoblock 96 thermocycler (Sensoquest, Germany). The resulting PCR products were separated by agarose gel electrophoresis $(2 \%$ agarose, w/v; at $80 \mathrm{~V}$ for 40 minutes) containing $\mathrm{SYBR}^{\circledR}$ Safe DNA gel stain (Thermo Fisher Scientific, USA) and visualized using the Gel DOC XR+ System Image Analyser (Bio-Rad, USA).

Table 1. The sequences and accession number of experimental primers used for conventional RT-PCR and 2-step RTqPCR.

\begin{tabular}{lllll}
\hline \multicolumn{1}{c}{ Genes } & \multicolumn{1}{c}{ Forward primer (5'-3') } & \multicolumn{1}{c}{ Reverse primer (5’-3') } & Accession number \\
\cline { 5 - 6 } GAPDH & GTCTTCACCACCATGGAGAAG & GCTTCACCACCTTCTTGATGTCATC & NM_0013579432 \\
$H M G C R$ & TTACTCCTTGGTGATGGGAGCTTG & TCCTGTCCACAGGCAATGTAGATG & NM_000859.3 \\
$L D L R$ & CAATGTCTCACCAAGCTCTG & TCTGTCTCGAGGGGTAGCTG & NM_001195799.2 \\
$A C A T 2$ & ACACCTCGATCTTGGTCCTGCCATA & GGAATGCAGACAGGGAGTCCT & NM_003578.4 \\
\hline
\end{tabular}


Gene expression analysis by 2-step real-time quantitative PCR (2-step RTPCR)

The expression of experimental genes was analyzed in Applied Biosystem ${ }^{\mathrm{TM}}$ StepOnPlus ${ }^{\mathrm{TM}}$ qPCR System (Thermo Fisher Scientific, USA) using the GoScript ${ }^{\mathrm{TM}}$ Reverse Transcription System (Promega, USA) and GoTaq ${ }^{\circledR}$ qPCR Master Mix (Promega, USA) according to the manufacturer's instructions. In brief, the cycling conditions were $95^{\circ} \mathrm{C}$ for 2 minutes followed by 40 cycles of denaturation $\left(95{ }^{\circ} \mathrm{C}, 3\right.$ seconds), annealing (GAPDH: $52.0^{\circ} \mathrm{C}, \mathrm{HMGCR}: 58.7^{\circ} \mathrm{C}$, LDLR: $64.0{ }^{\circ} \mathrm{C}$, and ACAT2: $57.7{ }^{\circ} \mathrm{C}, 15$ seconds) and extension $\left(60{ }^{\circ} \mathrm{C}, 1\right.$ minute). $G A P D H$ was used as the internal control, and the expression of experimental genes was calculated according to the $\Delta \Delta \mathrm{CT}$ method. Data were presented as fold-change to the control.

\section{Statistical analyses}

Data were analyzed by One-Way ANOVA test using the GraphPad Prism version 7 for Windows, GraphPad Prism (USA). Results were presented as mean \pm standard deviation and $P$-value $<0.05$ was considered as statistically significant.

\section{Results}

\section{Cell viability assay}

HepG2 cells were treated with RA $(0-10 \mu \mathrm{M})$ for 24 hours and the results were depicted in Figure 1. The trend of the results illustrated that cell viability decreases dose-dependently with RA incubation for 24 hours. Notably, the $\mathrm{IC}_{50}$ of RA was found to be at $27.72 \mu \mathrm{M}$. In line with the findings from this assay, $1 \mu \mathrm{M}$ and $5 \mu \mathrm{M}$ of RA were used to treat cells in the following experiments.

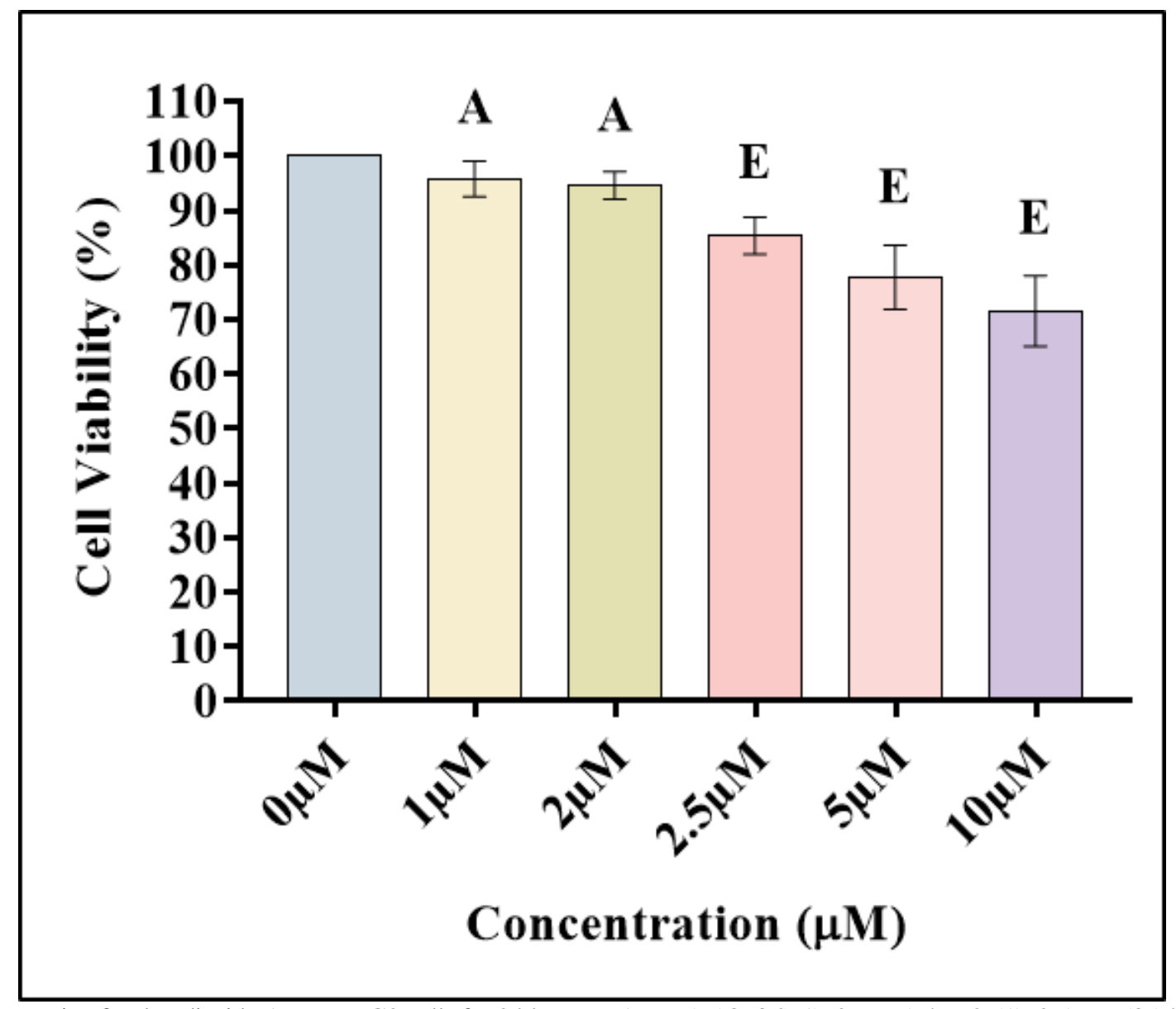

Fig. 1. Cytotoxic of Rebaudioside A on HepG2 cells for 24 hours at $1 \mu \mathrm{M}(95.8 \pm 3.271), 2 \mu \mathrm{M}(94.6 \pm 2.51), 2.5 \mu \mathrm{M}(85.4 \pm 3.362), 5$ $\mu \mathrm{M}(77.8 \pm 5.891)$ and $10 \mu \mathrm{M}(71.6 \pm 6.427)$ against control group $(0 \mu \mathrm{M})$. Graph followed by different upper case letter (A-E) represents a significant difference $(\mathrm{p}<0.05)$ treatment wise $(\mathrm{n}=5)$. 


\section{Cholesterol quantification and protein determination}

Data illustrated in Figure 2 indicated the extracted total cellular cholesterol (TCC) level in different groups of cells was considerably affected by treatments. According to the results, the highest value for TCC was recorded in HRA following $\mathrm{PC}>\mathrm{LRA}>\mathrm{NC}$, while the lowest was observed in BC. Surprisingly, treatment of cells with LRA has little to no effects on TCC as the result is almost similar to NC.

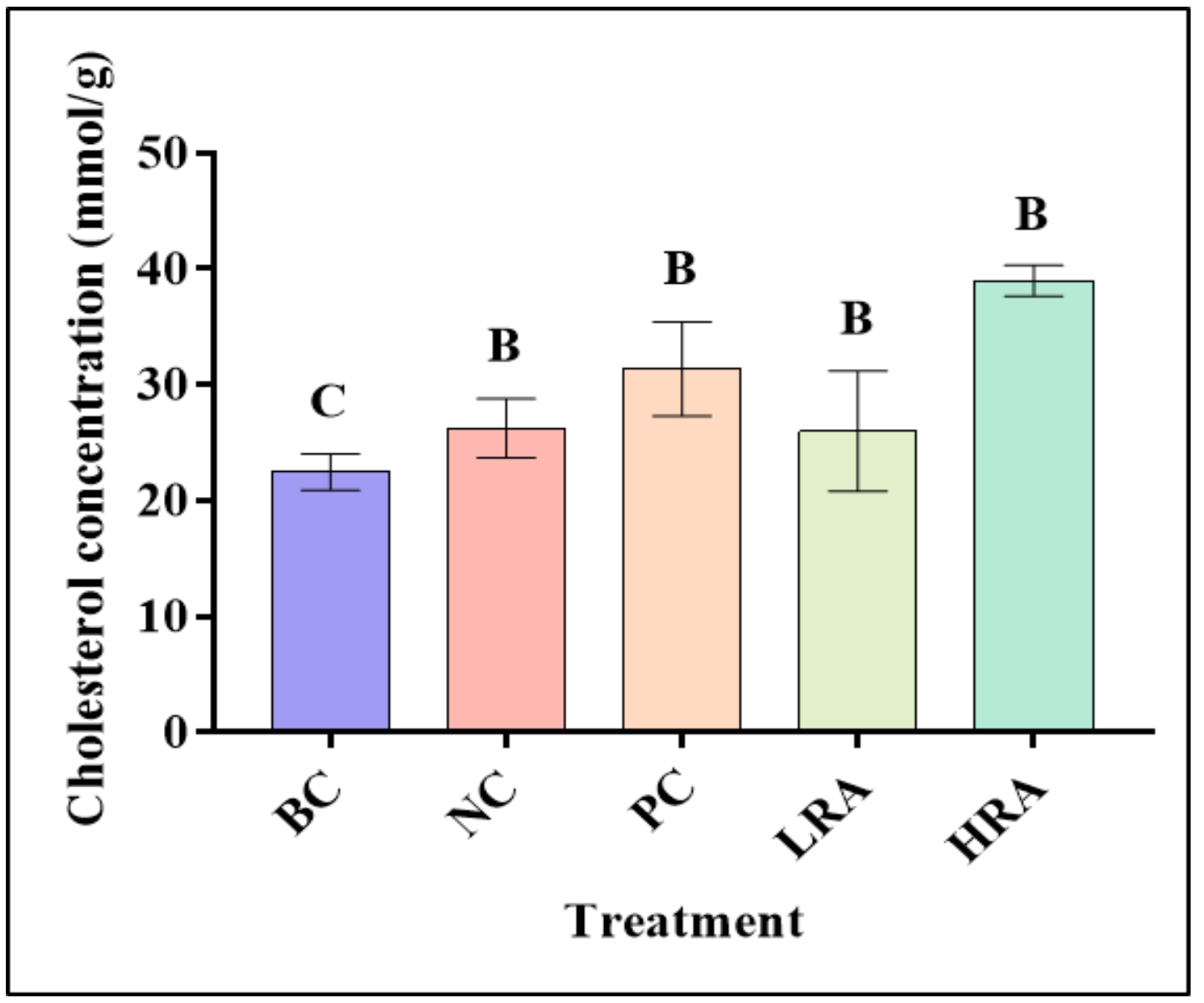

Fig. 2. TCC extracted from treated cells for 24 hours, BC (22.49 \pm 1.581$)$, NC (26.2 \pm 2.555$),$ PC (31.38 \pm 4.067$)$, LRA $(25.99 \pm 5.177)$ and HRA (38.98 \pm 1.318$)$. Graph followed by different upper-case letter (A-E) represents a significant difference $(\mathrm{p}<0.05)$ treatment wise $(n=5)$.

\section{Immunofluorescence microscopy}

To determine the expression of LDLR and LDs, a qualitative assessment by fluorescent staining was employed. The microphotographs depicted in Figure 3 suggest that HRA promoted the upregulation of LDLR expression on cells' surface causing higher intracellular lipid mobilization and further reserved into LDs. The expression of LDLR was qualitatively prominent in HRA> LRA> PC but absent in both $\mathrm{NC}$ and $\mathrm{BC}$. However, the presence or absence of LDLR does not affect LDs formation observed in all groups. It is important to highlight that no strong conclusions could be drawn based on the qualitative assessment of immunofluorescence staining although it reflects the results of the cholesterol quantification assay. Therefore, to ensure the molecular mechanism of RAinducing cellular uptake of cholesterol is properly understood, gene expression analysis was conducted. 

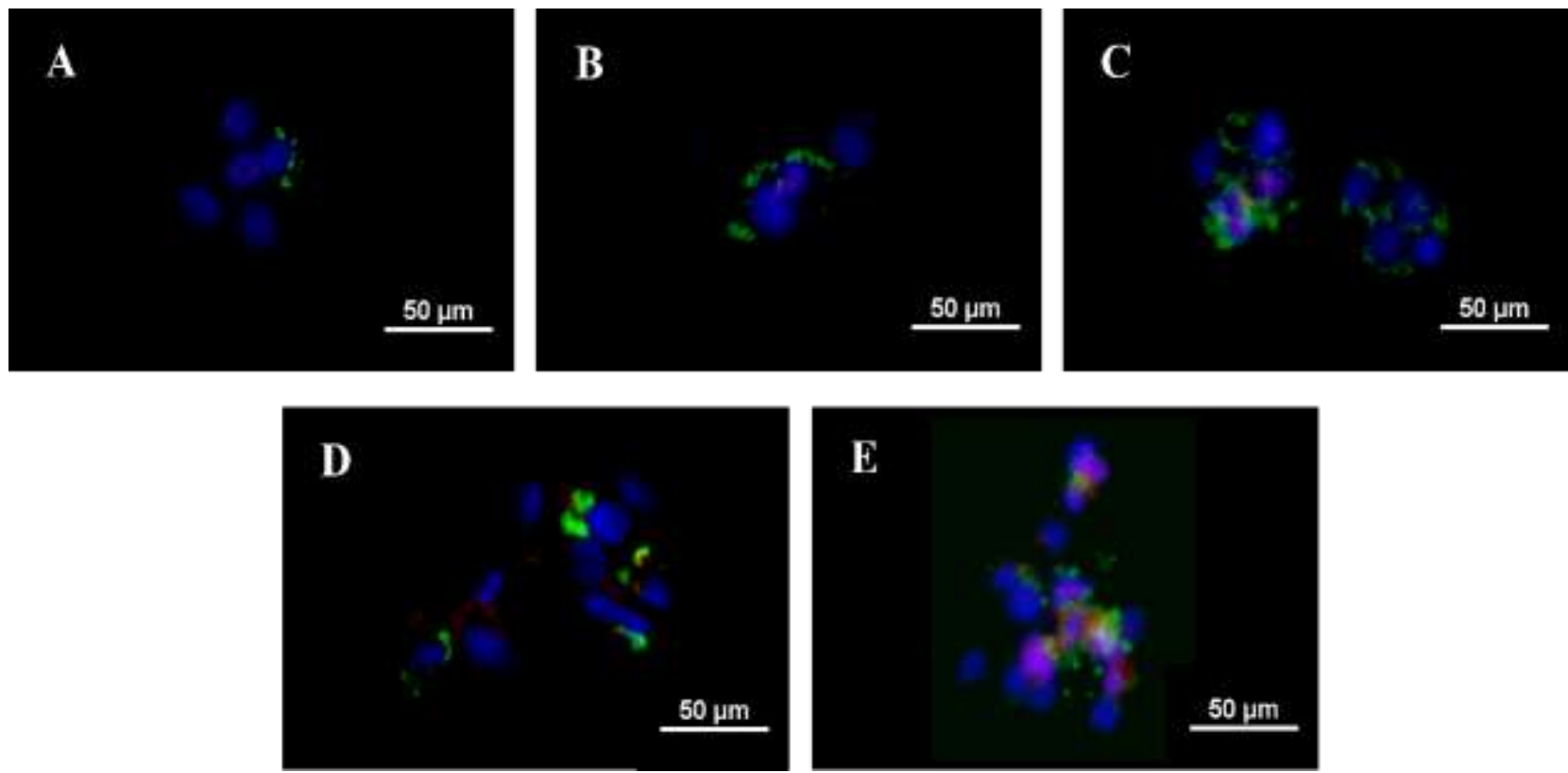

Fig. 3. Qualitative assessment of immunofluorescence microphotographs on treated cells for 24 hours (A; BC, B; NC, C; PC, D; LRA and E; HRA). Fluorescence staining was as follows: blue (nucleus), green (lipid droplets), and red (low-density lipoprotein receptor) with magnification at $200 X(n=4)$.

\section{Gene expression}

The purity of extracted RNA samples was in a range of 2.0 to 2.1 at an absorbance ratio of 260$280 \mathrm{~nm}$ wavelengths. The annealing temperature of each experimental and internal control gene was determined by conventional RT-PCR followed and qualitative assessment of bands via agarose gel electrophoresis (GAPDH: $490 \mathrm{bp}$, HMGCR: 747 bp, LDLR: 258 bp, and ACAT2:
$117 \mathrm{bp}$ ). The optimized annealing temperatures were adapted to cycling conditions for gene expression analysis by 2 -step RT-PCR. The expression of experimental genes was illustrated as expression-fold in Figure 4. It was found that HRA significantly down-regulated HMGCR expression and at the same time up-regulating $L D L R$ and ACAT2 genes as compared to other treatment groups.

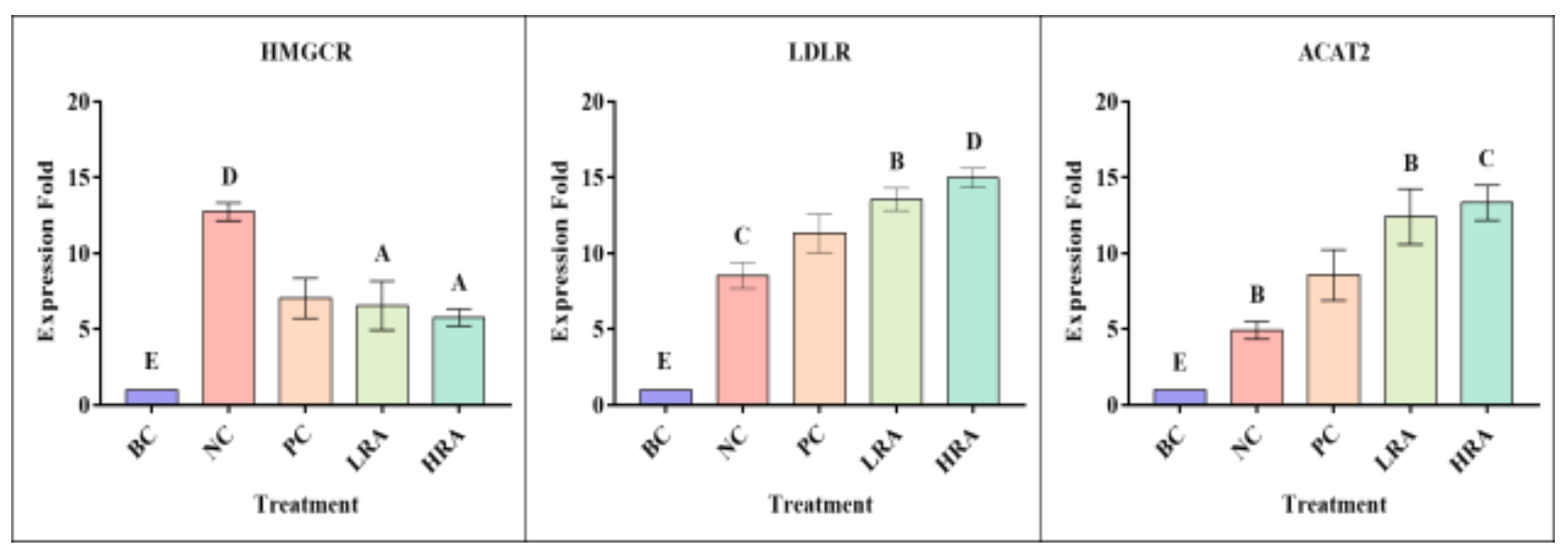

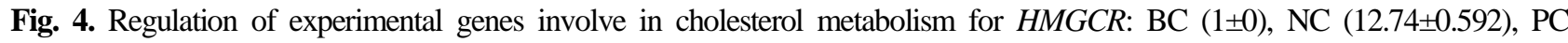

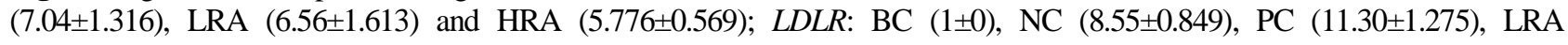
(13.56 \pm 0.748$)$ and HRA (14.96 \pm 0.640$)$; and ACAT2: BC (1 \pm 0$)$, NC (4.93 \pm 0.588$),$ PC (8.57 \pm 1.676$)$, LRA (12.43 \pm 1.839$)$ and HRA $(13.33 \pm 1.155)$. Graph followed by different upper-case letter $(A-E)$ represents a significant difference $(p<0.05)$ treatment wise $(n=3)$. 


\section{Discussion}

To our knowledge, this work is novel in describing the anti-hypercholesterolemia activity of RA and the potential molecular regulation of cholesterol levels in HepG2 cells. The cell line was used as an in-vitro model as it expresses multi-differentiated functions of the liver, especially in cholesterol metabolism (26, 27). Available data from this in-vitro study could provide scientific evidence on the application of RA therapeutical outcomes.

We first validated the cytotoxic effects of RA in HepG2 cell lines via MTT assay. The present experiment has illustrated three interesting findings. First, the incubation of cells with $1 \mu \mathrm{M}$ and $2 \mu \mathrm{M}$ of RA did not show any significant difference in cell viability compared to untreated cells $(0 \mu \mathrm{M})$. Second, a decrease in cellular viability with a significant difference was observed as the concentration of RA given to cells increased $(2.5-10 \mu \mathrm{M})$. Third, cellular proliferation was absent in all treatments which may suggest the antiproliferative effects of RA. Following the current findings, the toxicity effect of RA is observed to be cell dependant. The higher concentration of RA significantly decreased the cellular viability and proliferation of these cells due to membrane ruptures induced by high osmotic pressure (28).

Next, we examined the effects of RA in regulating intracellular cholesterol mobilization. Although cholesterol is important in maintaining cellular architecture, dynamics, and functions $(27,29)$ high circulating cholesterol levels are a predisposing factor of atherosclerotic cardiac disease $(27,30,31)$. Here we observed the TCC levels in the HRA group are the highest. The analysis presumed that HRA in this study is a potential candidate of cholesterol-lowering substitute but could not be definitively proven by comparing the TCC levels and fluorescence staining entirely. Therefore, to ensure the molecular mechanism is properly understood, gene expression analysis was done.

In this analysis, cells treated with HRA show promising results by significantly altered cholesterol-regulating genes (HMGCR, LDLR, and $A C A T 2$ ) in comparison to other treatment groups. The first experimental gene observed, $H M G C R$ was down-regulated in HRA further suggest that RA is a potential cholesterollowering alternative to simvastatin. This gene encodes the rate-limiting enzyme in endogenous cholesterol synthesis, the 3hydroxy-3-methylglutaryl coenzyme A reductase $(H M G C R)$ (32). Statins possess a similar structure as HMGCR which competitively inhibits the enzyme results in decrease cholesterol levels in cells (33). We postulated that RA exerts similar biochemical and physiological effects as statins. Treatment of cells with HRA also caused an up-regulation of $L D L R$ and ACAT2 gene expression. The mechanisms could be further elaborated by a brief understanding of the negative feedback mechanism evoked by strings of proteins interaction initiated by sterol regulatory element-binding protein (SREBP).

SREBP is a transcriptional factor protein that controls lipids synthesis in cells $(34,35)$. This protein is encoded by two genes (SREBF1: SREBP-1 and SREBF2: SREBP-2) that regulate cholesterol synthesis genes $(27,36)$. Among the existing isoforms, SREBP-2 is expressed ubiquitously and tightly regulated by cellular cholesterol levels $(34,35,37)$. SREBP-2 is present in the endoplasmic reticulum (ER) membrane as an inactive precursor bound to SREBP cleavage-activating protein (SCAP) (38). SCAP possesses a cholesterol-sensing domain that responds to intracellular cholesterol depletion causing translocation of SREBP-2 to the Golgi in a vesicle. Here, the Site- 1 and -2 proteases are cleaved which release the $\mathrm{NH}_{2}$-terminal transcription-activation domain of the SREBP-2. The $\mathrm{NH}_{2}$-terminal enter the nucleus binds to a sterol response element and ameliorates the expression of SREBP-2 targeted genes (34, 3739).

We also demonstrated that RA decreased the expression of $H M G C R$ and its transcription product, the $H M G C R$ enzyme. Inhibition of the enzyme results in alleviated intracellular 
cholesterol levels which initiate the proteolytic activation of SREBP-2 (33). The activation of SREBP-2 initiates the transcription of the $L D L R$ gene results in increased expression of LDLR on the cell surface. $L D L R$ gene encodes the genetic information required for LDLR synthesis involved in cellular uptake of circulating LDLC $(33,40)$. The regulation of LDLC is important in providing balance cholesterol homeostasis necessary to prevent certain cardiovascular diseases development (41). Circulating LDLC binds to LDLR and endocytosed through clathrin-mediated endocytosis. Through chains of enzymatic reactions, LDLR is recycled to the cell surface via an endocytic recycling compartment, whereas non-esterified cholesterol is hydrolyzed from LDLC by lysosomal acid lipase (LAL).

Next, we observed that the expression of the ACAT2 gene in HRA is significantly up- regulated in parallel to the expression of $L D L R$. $A C A T 2$ encodes the acyl-CoA:cholesterol acyltransferase (ACAT2), an enzyme that catalyzes the esterification of cholesterol (42). ACAT2 is one of the membrane-bound Oacyltransferase (MBOAT) families that plays important role in neutral lipid synthesis for lipid droplets biogenesis (43). This enzyme is primarily localized to the ER that utilizes nonesterified lipids (e.g. fatty acyl CoA and cholesterol) to form cholesterol esters which are reserved in between the leaflets of the ER bilayer and give rise to oil lens as the concentration increases $(43,44)$. Subsequently, LDs will bud off into the cytosol (43). LDs are highly dynamic intracellular organelles that prevent lipotoxicity and oxidative stress in cells by buffering potentially toxic lipids and proteins accumulation (45). Figure 5 depicted the proposed molecular pathway of RA in regulating the intracellular intake of LDLC.

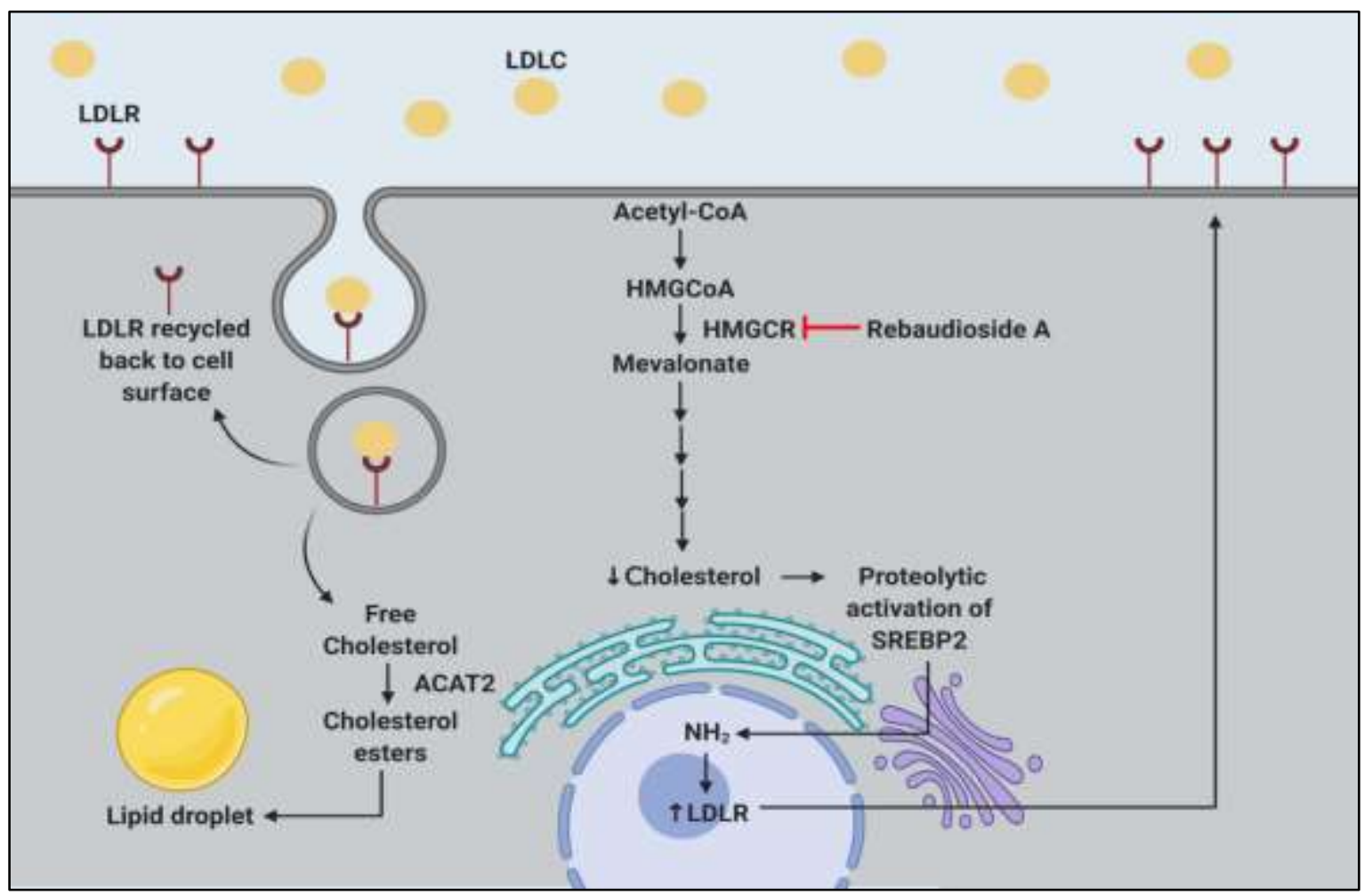

Fig. 5. A proposed molecular pathway of Rebaudioside A in ameliorating the expression of cholesterol-synthesis genes (HMGCR, $L D L R$ and ACAT2). Rebaudioside A competitively inhibits the enzymatic reaction of $H M G C R$ causing intracellular cholesterol depletion. Proteolytic activation of SREBP-2 was stimulated as the SCAP/SREBP-2 complex translocated to the Golgi followed by releasing of $\mathrm{NH}_{2}$-terminal into the nucleus. The consequences of this event lead to $L D L R$ gene transcription and more LDLR proteins exist on the cell surface. Circulating LDLC binds to LDLR and internalized via the clathrin-mediated endocytosis. In cells, LDLC segregates from its receptor (recycle to the surface) due to acidic $\mathrm{pH}$ and the former was hydrolysed by LAL. The non-esterified cholesterol molecules (free cholesterol) are toxic to cells, thereby esterified by ACAT2 protein into neutral lipids (cholesterol esters) and further stored in lipid droplets (This figure was created with BioRender.com). 
In summary, this novel in-vitro study shows that Rebaudioside A is a potential cholesterollowering candidate for statins. Considering its natural and non-cytotoxic characteristics, Rebaudioside A possesses few other therapeutical advantages to statins drawback. Despite the success demonstrated, this work is limited by its absence of other cholesterol synthesis genes namely SREPB-2, HMGCS, and $M V K$. Hence, future research should therefore seek to address this issue by implying these genes as well as other possible approaches such as large-scale multi-omics.

\section{References}

1. Sarker SD, Nahar L. Evidence-based phytotherapy: what, why and how?

Trends in phytochemical research. 2018;2(3):125-6.

2. Ahmad J, Khan I, Blundell R, Azzopardi J, Mahomoodally MF. Stevia rebaudiana Bertoni.: an updated review of its health benefits, industrial applications and safety. Trends in Food Science \& Technology. 2020;100:117-189.

3. Yadav SK, Guleria P. Steviol glycosides from Stevia: biosynthesis pathway review and their application in foods and medicine. Crit Rev Food Sci Nutr. 2012;52(11):988-98.

4. Mathur S, Bulchandani N, Parihar S, Shekhawat GS. Critical review on steviol glycosides: Pharmacological, toxicological and therapeutic aspects of high potency zero caloric sweetener. International Journal of Pharmacology. 2017;13(7):916-928.

5. Latarissa IR, Barliana MI, Lestari K. A Comprehensive Review of Stevia rebaudiana Bertoni effects on Human Health and Its Mechanism. Journal of Advanced Pharmacy Education and Research. 2020;10(2): 91-95.

6. Ullah A, Munir S, Mabkhot Y, Badshah SL. Bioactivity profile of the diterpene isosteviol and its derivatives. Molecules. 2019;24(4):678.

7. Chatsudthipong V, Muanprasat C. Stevioside and related compounds: therapeutic benefits beyond sweetness. Pharmacol Ther. 2009;121(1):41-54.
Regardless, the findings in this study provide a very useful insight to understand the mechanism of Rebaudioside A in lowering cholesterol levels.

\section{Acknowledgements}

Funding for this research was provided by Universiti Putra Malaysia (Geran Putra: GP/2020/9693000) and the Faculty of Veterinary Medicine (UPM). We thank Mr. Mohd. Jamil Samad for his assistance with fluorescence microscopy imaging. The authors declare they have no conflict of interests.

8. Saravanan R, Ramachandran V. Effect of Rebaudioside A, a diterpenoid on glucose homeostasis in STZ-induced diabetic rats. J Physiol Biochem. 2012;68(3):421-31.

9. Gupta E, Purwar S, Sundaram S, Rai GK. Nutritional and therapeutic values of Stevia rebaudiana: A review. Journal of Medicinal Plants Research. 2013;7(46):3343-3353.

10. Wang LS, Shi Z, Shi BM, Shan AS. Effects of dietary stevioside/rebaudioside $\mathrm{A}$ on the growth performance and diarrhea incidence of weaned piglets. Animal Feed Science and Technology. 2014;187:104-109.

11. Wang Y, Li L, Wang Y, Zhu X, Jiang M, Song E, et al. New application of the commercial sweetener rebaudioside a as a hepatoprotective candidate: Induction of the Nrf2 signaling pathway. Eur J Pharmacol. 2018;5(822):128-137. 12. Casas-Grajales S, Reyes-Gordillo K, CerdaGarcía-Rojas CM, Tsutsumi V, Lakshman MR, Muriel P. Rebaudioside A administration prevents experimental liver fibrosis: an in vivo and in vitro study of the mechanisms of action involved. J Appl Toxicol. 2019;39(8):1118-1131. 13. Saravanan R, Ramachandran V. Modulating efficacy of Rebaudioside A, a diterpenoid on antioxidant and circulatory lipids in experimental diabetic rats. Environ Toxicol Pharmacol. 2013;36(2):472-483.

14. Frostegård J. Immunity, atherosclerosis and cardiovascular disease. BMC medicine. 2013;11(1):1-3. 
15. Moriya J. Critical roles of inflammation in atherosclerosis. J Cardiol. 2019;73(1):22-27.

16. Deng W, Tang T, Hou Y, Zeng Q, Wang Y, Fan W, et al. Extracellular vesicles in atherosclerosis. Clin Chim Acta. 2019;495:109117.

17. Konstantinov IE, Jankovic GM. Alexander I. Ignatowski: a pioneer in the study of atherosclerosis. Tex Heart Inst J. 2013;40(3):246249.

18. Gratl V, Cheung RC, Chen B, Taghibiglou C, Van Iderstine SC, Adeli K. Simvastatin, an HMG-CoA reductase inhibitor, induces the synthesis and secretion of apolipoprotein AI in HepG2 cells and primary hamster hepatocytes. Atherosclerosis. 2002;163(1):59-68.

19. Cheraghi M, Ahmadvand H, Maleki A, Babaeenezhad E, Shakiba S, Hassanzadeh F. Oxidative stress status and liver markers in coronary heart disease. Rep Biochem Mol Biol. 2019;8(1):49-55.

20. Golomb BA, Evans MA. Statin adverse effects: A Review of the Literature and Evidence for a Mitochondrial Mechanism. Am $\mathrm{J}$ Cardiovasc Drugs. 2008;8(6):373-418.

21. Kitzmiller JP, Mikulik EB, Dauki AM, Murkherjee C, Luzum JA. Pharmacogenomics of statins: understanding susceptibility to adverse effects. Pharmgenomics Pers Med. 2016;9:97106.

22. Ramkumar S, Raghunath A, Raghunath S. Statin therapy: review of safety and potential side effects. Acta Cardiol Sin. 2016;32(6):631-639.

23. Sadeghi MM, Collinge M, Pardi R, Bender JR. Simvastatin modulates cytokine-mediated endothelial cell adhesion molecule induction: involvement of an inhibitory $G$ protein. $J$ Immunol. 2000;165(5):2712-8.

24. Mosmann T. Rapid colorimetric assay for cellular growth and survival: application to proliferation and cytotoxicity assays. J Immunol Methods. 1983;65(1-2):55-63.

25. Bligh EG, Dyer WJ. A rapid method of total lipid extraction and purification. Can J Biochem Physiol. 1959;37(8):911-7.

26. Donato MT, Tolosa L, Gómez-Lechón MJ. Culture and functional characterization of human hepatoma HepG2 cells. Methods Mol Biol. 2015;1250:77-93.
27. Afonso MS, Machado RM, Lavrador MS, Quintao EC, Moore KJ, Lottenberg AM. Molecular pathways underlying cholesterol homeostasis. Nutrients. 2018;10(6):760.

28. Wu X, Wang B, Chen T, Gan M, Chen X, Chen F, et al. The non-cytotoxicity characterization of rebaudioside $\mathrm{A}$ as a food additive. Food Chem Toxicol. 2014;66:334-40.

29. Goldstein JL, Brown MS. A century of cholesterol and coronaries: from plaques to genes to statins. Cell. 2015;161(1):161-172.

30. Gent J, Braakman I. Low-density lipoprotein receptor structure and folding. Cell Mol Life Sci. 2004;61(19-20):2461-70.

31. Cheraghi M, Shahsavari G, Maleki A, Ahmadvand H. Paraoxonase 1 activity, lipid profile, and atherogenic indexes status in coronary heart disease. Rep Biochem Mol Biol. 2017;6(1):1-7.

32. Ma S, Sun W, Gao L, Liu S. Therapeutic targets of hypercholesterolemia: HMGCR and LDLR. Diabetes Metab Syndr Obes. 2019;12:1543-1553.

33. Tirawanchai N, Homongkol $\mathrm{P}$, Chansriniyom C, Somkasetrin A, Jantaravinid J, Kengkoom K, et al. Lipid-lowering effect of Phyllanthus embilica and Alpinia galanga extracts on HepG2 cell line. PharmaNutrition. 2019;9:100153.

34. Shao W, Espenshade PJ. Expanding roles for SREBP in metabolism. Cell Metab. 2012;16(4):414-9.

35. Bhattacharya BS, Sweby PK, Minihane AM, Jackson KG, Tindall MJ. A mathematical model of the sterol regulatory element binding protein 2 cholesterol biosynthesis pathway. J Theor Biol. 2014;349:150-62.

36. Eberlé D, Hegarty B, Bossard P, Ferré P, Foufelle F. SREBP transcription factors: master regulators of lipid homeostasis. Biochimie. 2004;86(11):839-48.

37. Sato R. Sterol metabolism and SREBP activation. Arch Biochem Biophys. 2010;501(2):177-81.

38. Luo J, Yang H, Song BL. Mechanisms and regulation of cholesterol homeostasis. Nat Rev Mol Cell Biol. 2020;21(4):225-245.

39. van der Wulp MY, Verkade HJ, Groen AK. Regulation of cholesterol homeostasis. Mol Cell Endocrinol. 2013;368(1-2):1-16. 
40. K Jha C, Mir R, Elfaki I, Banu S, Chahal SM. Ldlr Gene Polymorphisms (Rs5925 and Rs1529729) Are Associated with Susceptibility to Coronary Artery Disease in a South Indian Population. Med Sci (Basel). 2019;7(7):80.

41. Zhang Y, Ma KL, Ruan XZ, Liu BC. Dysregulation of the low-density lipoprotein receptor pathway is involved in lipid disordermediated organ injury. Int $\mathbf{J}$ Biol Sci. 2016;12(5):569-79.

42. Parini P, Davis M, Lada AT, Erickson SK, Wright TL, Gustafsson U, et al. ACAT2 is localized to hepatocytes and is the major cholesterol-esterifying enzyme in human liver. Circulation. 2004;110(14):2017-23.

43. Wilfling F, Haas JT, Walther TC, Farese Jr RV. Lipid droplet biogenesis. Curr Opin Cell Biol. 2014;29:39-45.

44. Olzmann JA, Carvalho P. Dynamics and functions of lipid droplets. Nat Rev Mol Cell Biol. 2019;20(3):137-155.

45. Jarc E, Petan T. Focus: Organelles: Lipid Droplets and the Management of Cellular Stress. Yale J Biol Med. 2019;92(3):435-452. 